\title{
Intramolecular End-to-End Energy Transfer on Polypeptide Chains. Effects of Chain Length, Temperature, and Chain Stiffness ${ }^{\dagger}$
}

\author{
Masahiko Sisido, Syun Egusa,* Kazufumi Yagyu,* \\ and Yukio IMANISHI* \\ Research Center for Medical Polymers and Biomaterials, and \\ *Department of Polymer Chemistry, Kyoto University, \\ Kyoto 606, Japan
}

(Received August, 3, 1984)

\begin{abstract}
Static conformations of oligo- and polysarcosine chains were investigated by the energy transfer from an energy donating 2-naphthyl group attached to one end of the chain, to an energy accepting dimethylaminonaphthalenesulfonyl (dansyl) group on the other end of the chain [Dns-(Sar) ${ }_{n}-2 \mathrm{Nap}$ ]. The energy transfer efficiency $X$, was determined from fluorescence excitation spectra of the dansyl group as a function of chain length and temperature. An alternation in the transfer efficiency was observed with a step-by-step increase in the number of sarcosine units $n$, from 4 to 9 , with higher efficiencies for odd $n$ 's. The efficiency decreased with further increase in chain length but could still be detected at $\bar{n}=150(X=0.11)$. The transfer efficiency increased considerably at low temperatures and it approached unity at about $-60^{\circ} \mathrm{C}$, even for such long chains as $n=120$. The energy transfer between the donor-acceptor pair attached to a $\operatorname{rigid} \operatorname{poly}(\beta-$ benzyl L-aspartate) chain, Dns-[Asp $(\mathrm{OBzl})]_{n}-2 \mathrm{Nap}$, showed no temperature dependence. The marked enhancement observed on the polysarcosine chain was ascribed to a conformational change at low temperatures into folded or compact conformations.

KEY WORDS Energy Transfer / Intramolecular Interaction / Polysarcosine / Poly( $\beta$-benzyl L-aspartate) / Conformation /
\end{abstract}

Luminescence probe techniques have been used to study polymer conformations and chain dynamics in solution and in bulk. ${ }^{1}$ In the past decade, several workers prepared endcapped bichromophoric polymers and discussed their static ${ }^{2,3}$ and dynamic ${ }^{4-8}$ conformations. The end-capped systems are advantageous because end-to-end interactions can be selectively detected irrespective of the conformation in the rest of the polymer chain. In our previous communication, ${ }^{9}$ statics and dynamics of intramolecular excitation energy transfer in Dns-(Sar) ${ }_{n}$-2Nap polymers were studied, where the 2-naphthyl (2-Nap) and the dansyl (Dns) moieties function as an energy donor and energy acceptor, respectively. The transfer process consisted of two elementary processes, i.e., static and dynamic transfers. The static transfer occurs between an energy donor and energy acceptor in a closer region than a critical distance $r_{0}$ at the time of excitation. The dynamic transfer occurs for a pair of donor and acceptor well separated from each other at the time of excitation, and eventually become closer than $r_{0}$ by microBrownian chain motions during the lifetime of the excited state of the donor. Since the above study was carried out with polymer samples having a Poisson-type molecular weight distribution, the chain-length dependence in an oligomer region $(n=1-10)$ can not be discussed in detail.

Intramolecular end-to-end charge transfer (CT) interactions in oligosarcosines having an

\footnotetext{
† Part XIII in the series, Intrachain Reaction of a Pair of Reactive Groups Attached to Polymer Ends.
} 
electron donor ( $N, N$-dimethylaminoanilide) group at one end and an electron acceptor (3,5-dinitrobenzoyl) group at the other have also been studied as a function of the number of sarcosine units $n$ from 2 to $7 .^{10}$ The chainlength dependence of the CT association constant showed an alternating tendency with respect to $n$. The association constants were higher for even-membered oligomers than the nearest odd-membered ones. The specific chain-length dependence is quite interesting, since intramolecular reactions can be controlled by conformation of the intervening chain. Similar study using energy transfer probes was carried out and the results are described here. Since the effective radius for the energy transfer ( $>20 \AA$ ) is much longer than that for the CT interaction ( $c a .4 \AA$ ), a different chain-length dependence is expected. Intramolecular energy transfer has been studied for other $\operatorname{poly}(\alpha$-amino acid $)$ chains, ${ }^{11-19}$ but no such specific chain-length dependence has been reported so far.
In the present investigation, we also examined the environmental effects, i.e., temperature and solvent effects, on intramolecular interactions. If the energy transfer is an effective method to detect changes in the conformational equilibrium of polymers, this effectiveness should be most evident when the polymer conformation is changed by environmental factors. Hence, energy transfer was measured under different conditions in this study. Another important factor possibly influencing the intramolecular interactions is chain rigidity. The dynamic mechanism of energy transfer may be greatly suppressed when a polymer has a stiff chain as an $\alpha$-helix. Therefore, it should be worthwhile to compare intramolecular energy transfer efficiencies and their temperature dependence for flexible polysarcosine and rigid $\operatorname{poly}(\beta$-benzyl L-aspartate) chains. The steady-state of intramolecular energy transfers in Dns-(Sar) ${ }_{n}-2 \mathrm{Nap}$ (I) and Dns-[Asp $\left.(\mathrm{OBzl})\right]_{n}{ }^{-}$ $2 \mathrm{Nap}$ (II) system was studied, varying the

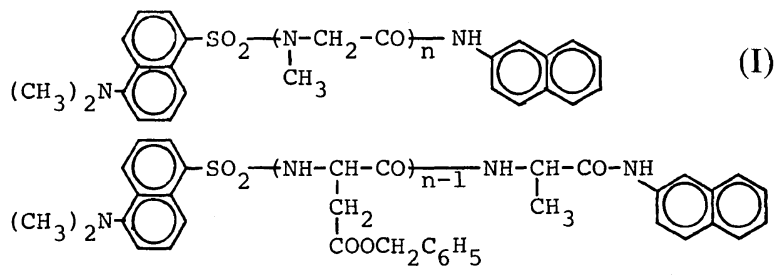

temperature from $40^{\circ} \mathrm{C}$ to $-60^{\circ} \mathrm{C}$ in ethanol and an ethanol/tetrahydrofuran mixture for I and in tetrahydrofuran for II. The oligomers of I were synthesized by NCA polymerization and polymer samples having a Poisson-type molecular weight distribution ${ }^{20}$ were fractionated by gel chromatography to isolate pure oligomers having definite number of sarcosine units.

\section{EXPERIMENTAL}

Synthesis and Fractionation of Dns-(Sar $)_{n^{-}}$ 2Nap and Dns-[Asp $(\mathrm{OBzl})]_{n}-2 \mathrm{Nap}$

Dns-(Sar) $)_{n}$-2Naps were prepared by the polymerization of sarcosine NCA with sarcosine-2-naphthylamide as an initiator, followed by the addition of dansyl chloride. ${ }^{9}$ These were fractionated by gel chromatography using a Sephadex LH-20 gel $(1.5 \mathrm{~cm}$ diameter and $190 \mathrm{~cm}$ length) equilibrated in ethanol. The chromatogram showed resolved peaks from $n=1$ to 13 and each fraction was again fractionated by the same column. The final chromatograms and peak areas used for subsequent experiments are shown in Figure 1.

Absorption spectra of the oligomers were virtually identical with each other and also indistinguishable from the sum of the spectra for the donor and acceptor model compounds, 


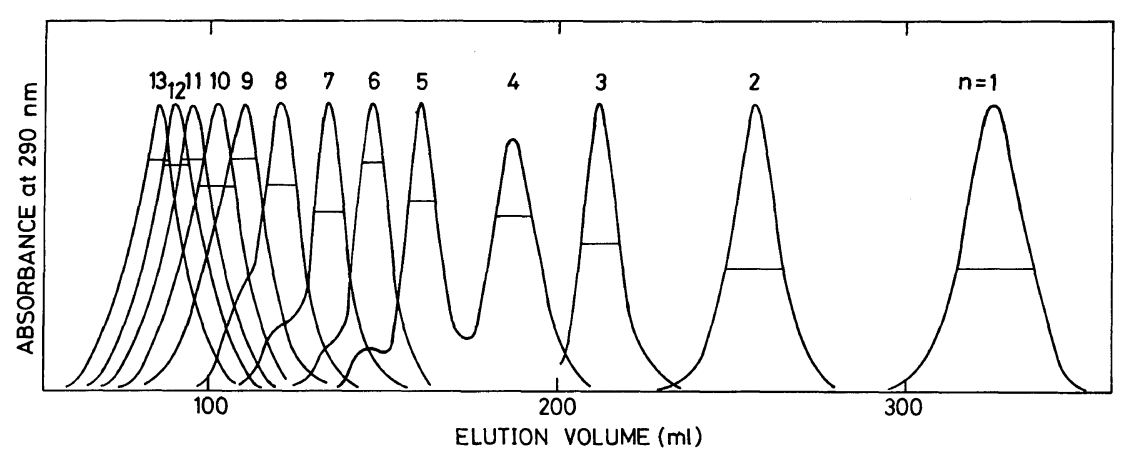

Figure 1. Gel permeation chromotograms of Dns-(Sar) ${ }_{n}-2 \mathrm{Nap}, n=1-13$ on Sephadex LH-20/ethanol column. Horizontal lines across the peaks indicate ranges used for energy transfer measurements.

indicating the absence of any ground-state interaction. Polymers having higher degrees of polymerization than 13 could not be fractionated into individual components and therefore those having the Poisson-type molecular weight distribution ${ }^{20}$ were used after purification by a Sephadex LH-60 column ( $1.5 \mathrm{~cm}$ diameter, $70 \mathrm{~cm}$ length) in ethanol. By the above procedure, oligomers with a definite number of sarcosine units $n$ from 1 to 13 and polymers having a Poisson-type molecular weight distribution with $n=20,30,40,60,85$, 120 , and 150 were obtained.

Polymers having no dansyl group, Ac(Sar) ${ }_{n}-2 \mathrm{Nap}$, were prepared by capping the $\mathrm{N}$ terminal with acetic anhydride instead of dansyl chloride and those having no energy donor group, Dns-(Sar) ${ }_{n}-\mathrm{NMe}_{2}$, were made by using sarcosine dimethylamide instead of sarcosine 2-naphthylamide as an initiator.

Synthesis of Dns-[Asp(OBzl) $]_{n}-2 \mathrm{Nap}$ (II) was also carried out by the NCA method. ${ }^{21} \mathrm{~L}-$ Alanine-2-naphthylamide (Sigma, recrystallized from ethanol) was used as an initiator and the polymers produced are more correctly designated as Dns-[Asp(OBzl) $]_{n-1}$-Ala-2Nap. Their molecular weight distribution estimated by fractionation with a Mercko-gel column in tetrahydrofuran, was very similar to that of the Poisson-type, and samples having the molecular weight distribution were used without fractionation.

\section{Measurements}

Fluorescence and fluorescence excitation spectra were recorded on a Hitachi MPF-4 spectrometer operated under an automatic correction mode. A variable-temperature quartz cell was used, which consists of a Dewar vessel with a quartz window. The cell temperature was measured by a thermocouple. The sample solution was degassed by bubbling nitrogen gas $(10 \mathrm{~min})$, followed by freeze-thaw cycles in a vacuum line.

The fluorescence decay was measured with a Hitachi time-resolved photometer equipped with an A-D converter. This machine was based on a gated sampling technique reported by Minami et al. ${ }^{22}$ Optical filters were used to select excitation $(255 \pm 25 \mathrm{~nm})$ and the emission $(310-390 \mathrm{~nm})$ wavelengths. The decay function was analyzed by a brute-force leastsquares fit, varying the fluorescence lifetime and a small drift in the time axis. The latter was necessary to obtain the best fit.

The concentration of the sample solution was adjusted to $1-5 \times 10^{-5} \mathrm{moldm}^{-3}$ with reference to the dansyl group $\left(\varepsilon_{340}=0.437 \times\right.$ $\left.10^{4}\right)$ or to 2-naphthyl group $\left(\varepsilon_{290}=0.595 \times 10^{4}\right)$ on the basis of the absorbance of the corresponding chromophores. At the above chromophore concentrations, no intermolecular energy transfer could be detected in the equimolar mixture of Ac-(Sar) $)_{40}-2 \mathrm{Nap}$ and Dns$(\mathrm{Sar})_{40}-\mathrm{NMe}_{2}$ over the temperature range of 
$-60^{\circ} \mathrm{C}-+30^{\circ} \mathrm{C}$. Ethanol and tetrahydrofuran as solvents for the fluorescence measurements were distilled over magnesium metal and $\mathrm{NaBH}_{4}$, respectively.

Absorption spectra were recorded on a Shimadzu UV-210 instrument, using a variable temperature cell with a $1 \mathrm{~cm}$ optical path.

Energy transfer was evident by the presence of a naphthyl peak in the fluorescence excitation spectrum $(270-350 \mathrm{~nm})$ of the bichromophoric oligomers monitored at the fluorescence maximum of the dansyl group $(520 \mathrm{~nm})$. The spectra for samples with different chain lengths were normalized at $350 \mathrm{~nm}$. The excitation spectra in the absence of an energy transfer were obtained with polymer samples without an energy donor. Since the spectra differed slightly when polymers of different degrees of polymerization were used, the average spectrum was used as that of the " $0 \%$-transfer." The absorption spectra of bichromophoric oligomers were averaged to obtain a " $100 \%$ transfer" excitation spectrum. Then, the transfer efficiency was calculated according to eq 1 .

$$
X=\frac{I-I_{0}}{I_{100}-I_{0}} \times 100(\%)
$$

where $I, I_{0}$, and $I_{100}$ represent intensities at the maximum excitation wavelength for the naphthyl group $(295 \mathrm{~nm})$ in the excitation spectra for $\mathrm{X} \%$-transfer, $0 \%$-transfer, and $100 \%$-transfer, respectively. When the temperature dependence of the transfer efficiency was examined, the $0 \%$ - and $100 \%$-transfer spectra were measured at each temperature. However, no significant difference was found in the two kinds of spectra over the temperature range between $+40^{\circ} \mathrm{C}$ and $-60^{\circ} \mathrm{C}$. Due to the uncertainty of the $0 \% \mathrm{o}^{-}$and $100 \%$ spectra, the absolute values of transfer efficiency include error of a few percent. But the profile of the efficiency-chain length plot or efficiency-temperature plot is insensitive to this uncertainty.
Evaluation of the Interaction Radius $r_{0}$

The interaction radius of the critical distance for the singlet-singlet energy transfer by the dipole-dipole mechanism, has been formulated by Forster ${ }^{23}$ as eq 2 . The

$$
r_{0}^{6}=\frac{9000(\ln 10) \kappa^{2} \eta_{\mathrm{D}}}{128 \pi^{2} N n^{4}} \int f(\tilde{v}) \varepsilon(\tilde{v}) \frac{\mathrm{d} \tilde{v}}{\tilde{v}^{4}}
$$

notations used in ref 23 are employed here. The orientation factor $\kappa^{2}$ was taken to be $2 / 3$. Thus $r_{0}$ was calculated from the quantum yield $\tau_{\mathrm{D}}$, and the fluorescence quantum spectrum of 2-naphthyl group covalently attached to polysarcosine chain [Ac-(Sar) $)_{n}-2 \mathrm{Nap}$ ], and the absorption spectrum of the dansyl group in Dns-(Sar) ${ }_{n}-\mathrm{NMe}_{2}$ at each temperarure.

\section{RESULTS}

\section{Energy Transfer Efficiency as a Function of Chain Length and Temperature}

The transfer efficiencies in Dns-(Sar) ${ }_{n}-2 \mathrm{Nap}$ are plotted as a function of the chain length $n$ in Figure 2. An alternating chain-length dependence with higher efficiency for odd-membered chains is seen at small $n$ 's. The efficiency decreased with an increase in chain length, but a detectable energy transfer $(11 \%)$ could still be observed for the longest chain $(\bar{n}=150)$ of Dns-(Sar) ${ }_{n}-2 \mathrm{Nap}$ at room temperarture. The temperature dependence of the transfer efficiency is shown in Figure 3. The efficiency increased at lower temperatures irrespective of chain length. The higher transfer efficiency at lower temperatures is an unexpected result, since the micro-Brownian motion which brings the two chromophores within the critical radius $r_{0}$ should become slower at lower temperatures.

To investigate this abnormal temperature effect in greater detail, the same pair of chromophores were attached to the ends of poly $(\beta$-benzyl L-aspartate) [Dns-[Asp$\left.(\mathrm{OBzl})]_{n}-2 \mathrm{Nap}\right]$ (II), whose polymer chain is much more rigid than the polysarcosine chain. The temperature dependence of the efficiency 


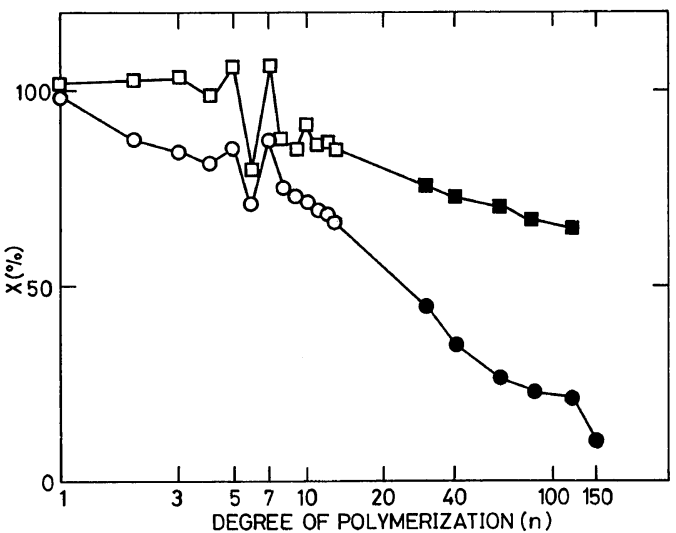

Figure 2. Chain-length dependence of energy transfer efficiencies for Dns-(Sar) $)_{n}-2 \mathrm{Nap}$ in ethanol at $24^{\circ} \mathrm{C}$ $(\bigcirc, \bigcirc)$, and $-20^{\circ} \mathrm{C}(\square, \square)$. Open circles and open squares represent data for samples with a definite number of sarcosine units. Filled circles and filled squares are for samples with Poisson-type molecular weight distribution.

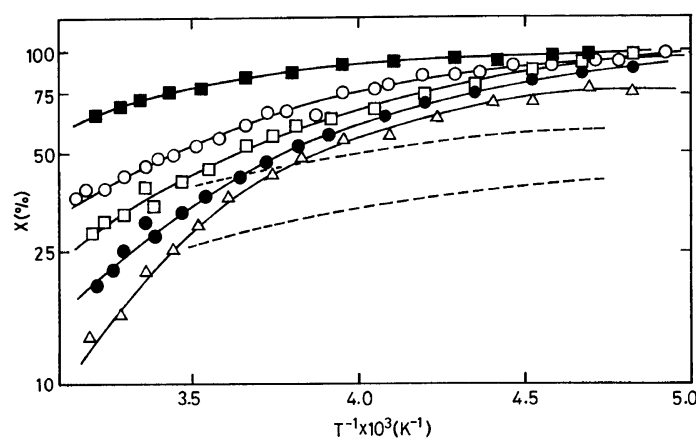

Figure 3. Temperature dependence of energy transfer efficiencies for Dns-(Sar) ${ }_{n}-2 \mathrm{Nap}$ in ethanol: $(\square), n=10$; $(\bigcirc), \bar{n}=30 ;(\square), \bar{n}=40 ;(\bigcirc), \bar{n}=60 ;(\triangle), \bar{n}=120$. The dashed lines represent theoretical temperature dependences for the static energy transfer for $n=11$ (upper line) and $n=15$ (lower line).

in Dns-[Asp(OBzl) $]_{n}-2 \mathrm{Nap}$ in tetrahydrofuran is shown in Figure 4. Contrary to the polysarcosine case, no temperature dependence was observed, indicating that the marked increase in efficiency observed with polysarcosine is related to flexibility of the polymer chain, by which formation of compact conformations at low temperatures is possible.

To assess the effect of solvent, tetrahy-

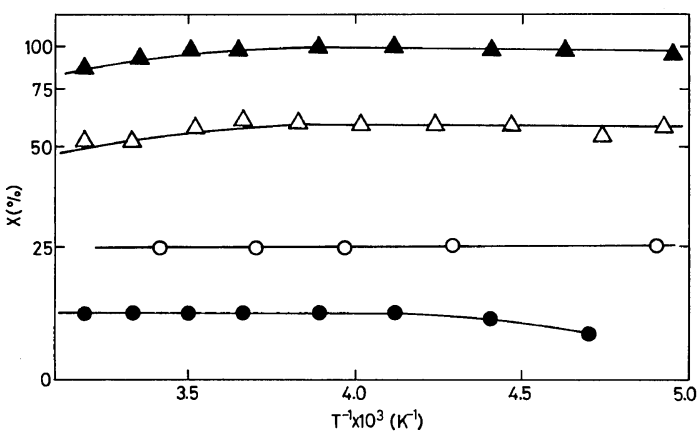

Figure 4. Temperature dependence of energy transfer efficiencies for Dns-[Asp(OBzl) $]_{n}-2 \mathrm{Nap}$ in tetrahydrofuran: $(\Delta), \bar{n}=6 ;(\triangle), \bar{n}=14 ;(\bigcirc), \bar{n}=26 ;(\bigcirc), \bar{n}=39$.

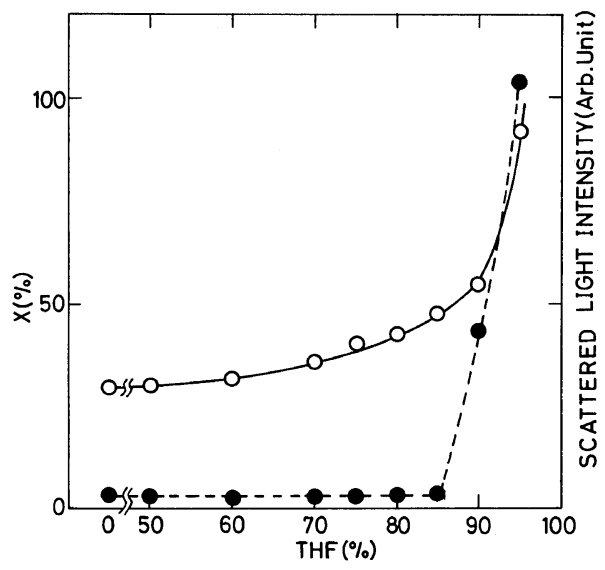

Figure 5. Solvent effect in energy transfer efficiencies for Dns-(Sar) ${ }_{60}-2 \mathrm{Nap}$ at room temperature in ethanol/ tetrahydrofuran mixture: $(\mathrm{O})$, energy transfer efficiencies; (O), intensity of scattered light at $300 \mathrm{~nm}$ detected vertically to the direction of incident light.

drofuran, a nonsolvent for polysarcosine, was added to an ethanol solution of Dns-(Sar) ${ }_{60}^{-}$ 2Nap. No detectable change was observed in either the absorption or fluorescence spectra. From the change in the fluorescence excitation spectrum, the energy transfer efficiency was calculated and plotted against solvent composition, as shown in Figure 5. In this figure, the intensity of scattered light from the sample solution is also given to show the onset of precipitation. It is clear that the efficiency begins to increase before the onset, indicating that the polymers begin to take compact con- 
formations before associating with each other. However, enhancement of the energy transfer by the addition of tetrahydrofuran was less pronounced than that observed by lowering the temperature.

\section{DISCUSSION}

\section{Chain-Length Dependence of Energy Transfer Efficiency}

The alternation observed in the chain-length dependence of the energy transfer in Dns(Sar) ${ }_{n}$-2Nap $(n=3-8)$ oligomers is particularly interesting. Since the distance between the two chromophores attached to the ends of the oligomers may be shorter ${ }^{24.25}$ than the cirtical distance $\left(r_{0}=c a .20 \AA\right.$, Table I $),{ }^{9}$ the chain length dependence should indicate orientational restriction of the rate of energy transfer. This sort of alternating orientational correlations between two terminal chromophores on oligosarcosines have been found experimentally and theoretically in the intramolecular charge-transfer (CT) complex formation on oligosarcosines having a terminal electron donor ( $N, N$-dimethylaminoanilide) group and a terminal electron acceptor (3,5-dinitrobenzoyl) group, A-(Sar) $)_{n}$-D (III). ${ }^{10}$

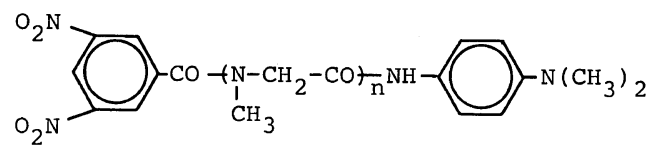

It is very strange that the alternation observed in the CT complex is opposite to that found with the energy transfer in the Dns(Sar) ${ }_{n}$-2Nap system, i.e., in the former case, the even-membered chains showed a higher intramolecular CT association constant, whereas in the latter case, the odd-membered chains show higher energy transfer efficiencies.

In the previous investigation of the conformational computation of $\mathrm{A}-(\mathrm{Sar})_{n}-\mathrm{D}$, the geometrical change in terminal chromophores was found to reverse the alternation in the intramolecular CT complex formation. ${ }^{10}$ If one assumes that the dihedral angle $\phi_{\mathrm{A}}$ be-

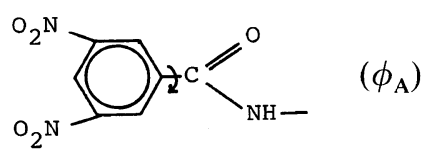

tween the planes of the dinitrobenzene ring and neighboring amide group is $90^{\circ}$, the alternation such that CT complex is formed more easily with chains having even $n$ 's was predicted and was actually observed in the CT complex case. But if $\phi_{\mathrm{A}}$ is assumed to be $26^{\circ}$, an inverse alternation was predicted. Therefore, the inverse alternations observed in the CT interaction and the energy transfer cases may result from different dihedral angles for $\phi_{\mathrm{A}}$ and $\phi_{\mathrm{Dns}}$, the latter being the dihedral angle between the planes of the dimethylaminonaphthyl group and the sulfamide group. It is plausible that $\phi_{\mathrm{A}}$ is near $90^{\circ}$ whereas $\phi_{\text {Dns }}$ is around $0^{\circ}$.

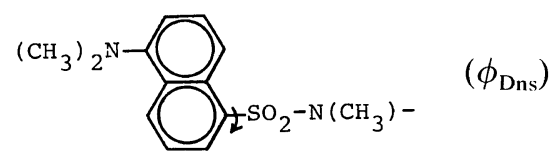

It should be noted that the energy transfer rate does not depend directly on the relative orientation of the two chromophores, but on that of the emission transition moment of the donor and the absorption transition moment of the acceptor. However, the emission and absorption transitions of naphthyl and dansyl groups have partially forbidden characters and have different polarizations within the aromatic planes for different vibronic transitions. ${ }^{18}$ The above discussion assumes nearly random orientation of the two transition moments within the aromatic planes.

\section{Temperature Dependence of the Transfer Ef- ficiency}

As can be seen in Figure 3, the energy transfer efficiency increases markedly with lowering the temperature. Since energy transfer is possible through two different mecha- 
nisms, i.e., static and dynamic transfers, the temperature effect on each process should be considered separately. It should be noted that although $r_{0}, \tau_{\mathrm{D}}$ (donor fluorescence lifetime), and $\eta_{\mathrm{D}}$ (donor fluorescence quantum yield) depend on temperature, the intrinsic rate constant of energy transfer $k_{\mathrm{t}}$ does not, provided the overlap integral of the donor fluorescence spectrum and acceptor absorption spectrum remain unchanged, as was observed in the case of dansyl/2-naphthyl pair. In the expression, $k_{\mathrm{t}}=\tau_{\mathrm{D}}{ }^{-1}\left(r_{0} / r\right)^{6}$, the increase in $r_{0}$ at low temperatures should be cancelled out by the increase in $\tau_{\mathrm{D}}$. The static transfer efficiency for the spatially fixed donor-acceptor pair increases at low temperatures due to the increase in the donor lifetime or alternatively, in $r_{0}$. These two expressions are equivalent. Therefore, for the static energy transfer in bichromophoric polymers having distributions of the chromophore-chromophore distances, it is sufficient to consider only two temperature-dependent factors, the end-to-end distance distribution and $r_{0}$.

For the dynamic transfer, the increase in $\tau_{\mathrm{D}}$ leads to an increase in the mean-square displacement as described below and its influence on the energy transfer should be considered separately from that by the increase in $r_{0}$.

Obviously, the micro-Brownian motion is suppressed at low temperatures and the contribution of dynamic transfer is reduced. But this may be compensated for by the increase in donor fluorescence lifetime. The overall balance of the two oppositely working effects can be roughly estimated by representing the mean-square displacement distance as, ${ }^{26}$

$$
\left\langle\Delta r^{2}\right\rangle=6 D \tau_{\mathrm{D}} .
$$

where $D$ is the diffusion coefficient of the donor moiety. The temperature dependence of $D$ may be roughly estimated from the Stodes equation,

$$
D=k T / 6 \pi a \eta
$$

The temperature dependence of the viscosity of ethanol is given in the literature $(\eta=8.41 \mathrm{cP}$ at $-60^{\circ} \mathrm{C}$ and 1.47 at $\left.+10^{\circ} \mathrm{C}\right){ }^{27}$ The donor lifetime was measured for the Ac-(Sar) ${ }_{10}-2 \mathrm{Nap}$ oligomer and is shown in Table I. The net temperature dependence of the mean displacement distance was estimated using these data and found to be positive; thus the decrease in temperature should descrease the dynamic transfer:

$$
\left[\left\langle\Delta r^{2}\right\rangle\left(10^{\circ} \mathrm{C}\right)\right] /\left[\left\langle\Delta r^{2}\right\rangle\left(-60^{\circ} \mathrm{C}\right)\right]=3.5 .
$$

The marked increase in the overall tranasfer efficiency observed at low temperatures may be accounted for by the increase in static transfer efficiency which overcomes the decrease in dynamic transfer.

As discussed above, only two factors should be considered for the enhancement of static transfer at low temperatures: the increase in

Table I. Temperature dependence of the fluorescence lifetime $\tau_{\mathrm{D}}$, and quantum yield $\eta_{\mathrm{D}}$, for the terminal 2-naphthyl group and

\begin{tabular}{|c|c|c|c|}
\hline Temperature & $\tau_{\mathrm{D}}$ & & $r_{0}$ \\
\hline${ }^{\circ} \mathrm{C}$ & $\mathrm{ns}^{\mathrm{a}}$ & & $\AA^{c}$ \\
\hline 10 & 6.5 & 0.055 & 18.7 \\
\hline 0 & 7.1 & & \\
\hline-10 & 8.5 & & \\
\hline-20 & 10.0 & 0.097 & 20.5 \\
\hline-30 & 10.9 & & \\
\hline-40 & 11.9 & & \\
\hline-50 & 13.6 & & \\
\hline-60 & 14.2 & 0.173 & 22.6 \\
\hline
\end{tabular}
interaction radius $r_{0}$, for the 2Nap/Dns pair in ethanol

a Average of lifetimes for Ac-(Sar $)_{n}-2 \mathrm{Nap}, \bar{n}=10,20$, and 40 .

${ }^{\text {b }}$ Quantum yield of Ac-(Sar $)_{10}-2$ Nap.

c Interaction radius calculated from the absorption spectrum of Dns-(Sar) ${ }_{10}$-DMA and fluorescence spectrum of Ac-(Sar $)_{10}-2$ Nap. The refractive index of solvent (ethanol) was taken to be $1.38\left(330 \mathrm{~nm}, 20^{\circ} \mathrm{C}\right.$, "International Critical Tables of Numerical Data, Physics, Chemistry, and Technology," McGraw Hill, 1926). The temperature dependence of the refractive index, which was found to decrease the $r_{0}$ value at $-60^{\circ} \mathrm{C}$ by about $2 \%$, was neglected. 
the interaction radius $r_{0}$ and change in the polysarcosine conformation to increase the population of compact conformations favorable for energy transfer. $r_{0}$ values at different temperatures were calculated from the fluorescence spectrum of the donor and absorption spectrum of the acceptor, using eq 2. The results are shown in Table I.

To evaluate the contribution of increase in $r_{0}$ to that in static energy transfer efficiency at low temperatures, a theoretical calculation of the static transfer efficiency according to eq 5 was carried out.

$$
X=\int_{0}^{\infty} \frac{r_{0}^{6}}{r_{0}^{6}+r^{6}} W(r) \mathrm{d} r
$$

The temperature-independent end-to-end distribution function $W(r)$, was taken from a previous Monte Carlo calculation for selfavoiding polysarcosine chains ${ }^{24.25}$ and the temperature dependent $r_{0}$ value was taken from Table I. The results for polysarcosine chains with $n=11$ and 15 are shown by dashed curves in Figure 3. It is clear that the effect of the temperature dependence of $r_{0}$ is relatively small, although it cannot be neglected.

Thus, it is concluded that the marked increase in overall efficiency is mainly due to the shift in conformational equilibrium to favor compact conformations in which the energy transfer occurs efficiently. This is supported by the absence of temperature dependence in Dns-[Asp(OBzl) $]_{n}-2 \mathrm{Nap}$ polymers, whose main chain is too stiff for such a conformational change to occur. The increase in compact or folded conformations has been suggested from the temperature dependences of an intramolecular chemical reaction in aqueous solution ${ }^{28}$ and an intramolecular CT interaction in ethanol or chloroform solution ${ }^{10.29}$ on poly- or oligosarcosines.

It is well known that some polymer chains reduce their dimensions at low temperatures. ${ }^{30}$ For example, the temperature derivative of the logarithms of the mean-square end-to-end distance, $\mathrm{d}\left(\ln \left\langle r^{2}\right\rangle\right) / \mathrm{d} T$, is positive for poly- (oxyethylene) $\left(0.23 \times 10^{-3} \mathrm{deg}^{-1}\right)$ and for poly(dimethylsiloxane) $\quad\left(0.75 \times 10^{-3} \mathrm{deg}^{-1}\right)$. However, the temperature dependences are much smaller than that for polysarcosine. In the case of poly(dimethylsiloxane), the temperature coefficient corresponds to the decrease in its mean end-to-end distance from $40.0 \AA$ to $38.5 \AA$ by a temperature decrease of $100^{\circ}$, which presumably would not be detected by an energy transfer experiment. The marked temperature dependence of polysarcosine conformations should thus be given special attention.

It should be noted that the compact or folded conformation does not imply any regular or helical conformation of polysarcosine. Conformation of polysarcosine is random even at low temperatures as evident from the coexistence of cis and trans amide bonds in the NMR spectra. ${ }^{31}$

The conformational change can also be seen by adding a nonsolvent (tetrahydrofuran) to ethanol solution of polysarcosine (Figure 5). The energy transfer efficiency increases before the onset of intermolecular association. However, the solvent effect is rather small, compared to the temperature dependence.

\section{REFERENCES}

1. Y. Nishijima, J. Polym. Sci., C, 31, 353 (1970).

2. Y. Imanishi, J. Polym. Sci., Macromol. Rev., 14, 1 (1979).

3. M. A. Winnik, Chem. Rev., 81, 491 (1981).

4. K. Horie and I. Mita, Kobunshi, 27, 637 (1978).

5. K. Horie, Kobunshi, 32, 196 (1983).

6. M. A. Winnik, A. E. C. Redpath, K. Paton, and J. Danhelka, Polymer, 25, 91 (1984).

7. C. Cuniberti and A. Perico, Eur. Polym. J., 13, 369 (1977).

8. T. Kanaya, K. Goshiki, M. Yamamoto, and Y. Nishijima, J. Am. Chem. Soc., 104, 3580 (1982).

9. M. Sisido, Y. Imanishi, and T. Higashimura, Macromolecules, 12, 975 (1979).

10. (a) M. Sisido, Y. Kanazawa, and Y. Imanishi, Biopolymers, 20, 653 (1981); (b) M. Sisido and Y. Imanishi, Biopolymers, 20, 665 (1981).

11. L. Stryer and R. P. Hargland, Proc. Natl. Acad. Sci. U.S.A., 58, 719 (1967).

12. G. Gabor, Biopolymers, 6, 809 (1968). 
13. R. Guillard, M. Leclerc, A. Loffet, J. Leonis, B. Wilmet, and A. Englert, Macromolecules, 8, 134 (1975).

14. R. Guillard and A. Englard, Biopolymers, 15, 1301 (1976).

15. M. Leclerc, S. Premilat, and A. Englert, Biopolymers, 17, 2459 (1978).

16. H. C. Chiu and R. Bersohn, Biopolymers, 16, 277 (1977).

17. E. Haas, M. Wilchek, E. Katchalski-Katzier, and I. Z. Steinberg, Proc. Natl. Acad. Sci. U.S.A., 72, 1807 (1975).

18. E. Haas, E. Katchalski-Katzier, and I. Z. Steinberg, Biopolymers, 17, 11 (1978).

19. J. Eisinger, B. Feuer, and A. A. Lamola, Biochemistry, 8, 3908 (1969).

20. M. Sisido, Y. Imanishi, and T. Higashimura, Makromol. Chem., 178, 3107 (1977).

21. M. Sisido, K. Yagyu, H. Kawakubo, and Y. Imanishi, Int. J. Biol. Macromol., 4, 313 (1982).

22. S. Minami, T. Uchida, and S. Fujita, Oyo butsuri, 37, 34 (1968).
23. N. Mataga and T. Kubota, "Molecular Interactions and Electronic Spectra," Marcel Dekker, New York, N.Y., 1970, Chapter 5.

24. M. Sisido, Y. Imanishi, and T. Higashimura, Macromolecules, 9, 320 (1976).

25. M. Sisido, Y. Imanishi, and T. Higashimura, Macromolecules, 9, 389 (1976).

26. S. Chandrasekhar, Rev. Mod. Phys., 15, 1 (1943).

27. R. Weast, Ed., "Handbook of Chemistry and Physics," 57th ed, CRC Press, Cleveland, Ohio, 1976, p F-54.

28. M. Sisido, T. Mitamura, Y. Imanishi, and $T$. Higashimura, Macromolecules, 9, 316 (1978).

29. (a) M. Sisido, H. Takagi, Y. Imanishi, and T. Higashimura, Macromolecules, 10, 125 (1977); (b) H. Takagi, M. Sisido, Y. Imanishi, and T. Higashimura, Bull. Chem. Soc. Jpn., 50, 807 (1977).

30. P. J. Flory, "Statistical Mechanics of Chain Molecules," Wiley, New York, N.Y., 1969, Chapter 5.

31. (a) M. Sisido, Y. Imanishi, and T. Higashimura, Biopolymers, 11, 399 (1972); (b) unpublished result. 